\title{
Phenotypic and Genotypic Diversity of Nosocomial Multi-Drug Resistant Klebsiella pneumoniae Isolated from Cancer Patients in Cairo, Egypt
}

\author{
Mahmoud M. Tawfick ${ }^{1,2 *}$, Samira M. Hamed ${ }^{2}$, Hadeel M. Darwich ${ }^{2}$ and \\ Hadir A. El-Mahallawy ${ }^{3}$ \\ ${ }^{1}$ Microbiology and Immunology Department, Faculty of Pharmacy, \\ Al-Azhar University, Cairo, Egypt \\ ${ }^{2}$ Microbiology and Immunology Department, Faculty of Pharmacy, October University for \\ Modern Sciences and Arts (MSA), Cairo, Egypt \\ ${ }^{3}$ Clinical Pathology, Medical Oncology Department, National Cancer Institute, \\ Cairo University, Egypt \\ *Corresponding author
}

\begin{tabular}{|c|c|}
\hline \multirow{6}{*}{$\begin{array}{l}\text { Ke y w o r d s } \\
\text { Klebsiella } \\
\text { pneumoniae, } \\
\text { RAPD-PCR, } \\
\text { multi-drug } \\
\text { resistant, typing, } \\
\text { genetic diversity. }\end{array}$} & A B S T R A C T \\
\hline & \multirow{10}{*}{$\begin{array}{l}\text { Nosocomial Klebsiella pneumoniae infections are particularly a problem among } \\
\text { cancer and immunocompromised patients worldwide. K. pneumoniae strains are } \\
\text { widespread in nature thus typing is required to discriminate them in the } \\
\text { epidemiological investigations. In this study, the diversity of } 43 \text { nosocomial multi- } \\
\text { drug resistant (MDR) K. pneumoniae isolates recovered from different clinical } \\
\text { specimens collected from cancer patients at National Cancer Institute, Cairo, } \\
\text { Egypt, were phenotypically and genotypically analysed. These isolates were } \\
\text { identified using conventional microbiological methods and the API } 20 \mathrm{E} \text { system. } \\
\text { Investigation of the antimicrobial susceptibility patterns against } 16 \text { diverse } \\
\text { antimicrobial agents revealed that all isolates are MDR. The phenotyping was } \\
\text { performed using the API 20E-based biotyping and antibiogram typing which } \\
\text { showed three different biotypes and } 25 \text { antibiogram types among the isolates. The } \\
\text { genotyping using random amplified polymorphic DNA (RAPD) analyses revealed } \\
39 \text { different RAPD-based fingerprints and/or } 43 \text { different patterns among the } \\
\text { isolates. In conclusion, K. pneumoniae infections in this institution have been } \\
\text { caused by diverse MDR } K \text {. pneumoniae genotypes and/or phenotypes clone groups } \\
\text { with isolates in the same phenotype group possess different genotypes. Biotyping } \\
\text { and antibiogram typing of } K \text {. pneumoniae isolates have been shown to be well for } \\
\text { preliminary screening of strain relatedness. The use of RAPD-PCR-based analyses } \\
\text { is recommended, which has high discriminatory power providing definite } \\
\text { information to evaluate the epidemic status of the nosocomial infections caused by } \\
\text { MDR K. pneumoniae. }\end{array}$} \\
\hline & \\
\hline & \\
\hline & \\
\hline & \\
\hline Article Info & \\
\hline & \\
\hline & \\
\hline & \\
\hline & \\
\hline
\end{tabular}

\section{Introduction}

$K$. pneumoniae, a member of the Enterobacteriaceae family, is one of the most common opportunistic Gram-negative pathogens (Zhao et al., 2010). This bacterium has emerged worldwide as a 
leading cause of nosocomial infections, including pneumonia, bacteremia, urinary tract infections and wound infections (Cao et al., 2015). K. pneumoniae infections are particularly a problem among the elderly, the immunocompromised persons and patients with underlying malignancy (Henao-Martínez et al., 2013; Holt et al., 2015). The main nosocomial reservoirs of $K$. pneumoniae include contaminated medical equipments, hands of hospital staff and the gastrointestinal tract of patients (Samra et al., 2007).

At present, the emergence of MDR $K$. pneumoniae strains represents an urgent important threat to human health; leaving only limited options for treatment. Thus, infections with MDR $K$. pneumoniae strains are usually associated with high morbidity and mortality, long hospital stay and high healthcare costs (Cao et al., 2015; Passet and Brisse, 2015). All mechanisms of antimicrobial resistance demonstrated in Gram-negative bacteria have been mostly manifested in $K$. pneumoniae, such as enzymatic hydrolysis, target mutation and reduced intracellular accumulation through reduced uptake and active efflux (Filgona et al., 2015). Besides extended-spectrum $\beta$ lactamase (ESBL) production, $K$. pneumoniae is frequently known to be resistant to multiple antimicrobial agents including fluoroquinolones, aminoglycosides and trimethoprim/ sulfamethoxazole (Tan et al., 2015). Considerably, the acquisition of carbapenemases-coding genes has depleted the last choice for treating infections caused by MDR K. pneumoniae (He et al., 2015).

Bacterial typing, including phenotyping and genotyping, are used for detecting the diversity among strains of the same species. Phenotyping is mainly based on the different biochemical reactions, serological reactions and antimicrobial susceptibility profiles. On the other hand, genotyping refers to the discrimination of bacterial strains based on their genetic construction (Li et al., 2009). Importantly, several outbreaks of infection caused by MDR $K$. pneumoniae strains have been reported (Cartelle et al., 2004). In addition, various typing methods have been applied to recognize the transmission patterns for surveillance and prevention of the dissemination of MDR $K$. pneumoniae in a hospital setting. Pulsed-field gel electrophoresis (PFGE) analysis of genome has been shown to be a leading discriminatory technique for typing; however, it is technically demanding, timeconsuming and requires specific equipment. Consequently, there is a need for less expensive and laborious methods that allow rapid evaluation of the relatedness of strains, on a local scale, to identify outbreaks with MDR $K$. pneumoniae. In recent studies, PCR-based typing techniques, such as randomly amplified polymorphic DNA (RAPD) analysis, and enterobacterial repetitive intergenic consensus sequence PCR (ERIC-PCR) which are faster and easier to perform, have been successfully used for typing $K$. pneumoniae isolates (Cartelle et al., 2004; Sachse et al., 2014; Ashayeri-Panah et al., 2014).

This study aimed to investigate the diversity among MDR $K$. pneumoniae isolated from different clinical specimens collected from cancer patients to determine the epidemiological status of this organism at National Cancer Institute, Cairo, Egypt.

\section{Materials and Methods}

\section{Isolation, identification and biotyping of $K$. pneumoniae isolates}

A total of 43 non-duplicated $K$. pneumoniae isolates were included in this study. These 
isolates were recovered from various clinical specimens including blood (27), pus (10), sputum (4), urine (1) and stools (1), collected from cancer inpatients at National Cancer Institute, Cairo, Egypt, during the period from September 2015 to January 2016. Isolates were identified to species level using conventional microbiological methods, such as cultural characteristics on MacConkey's agar, Gram staining and biochemical testing. Identification was confirmed using the API 20E system (BioM'erieux, France). Both Luria-Bertani broth (LB) and agar (Lab M, UK) were used for growing isolates at $37^{\circ} \mathrm{C}$. The isolates were subjected to biotyping on the basis of the biochemical profile produced by the API $20 \mathrm{E}$ system following the manufacturer's instructions.

\section{Antimicrobial susceptibility testing and antibiogram typing}

Antimicrobial susceptibilities of $K$. pneumoniae isolates to different antimicrobial agents were determined using Kirby-Bauer disk diffusion method on Mueller-Hinton agar following the Clinical and Laboratory Standards Institute (CLSI, 2015) guidelines. A number of 16 antimicrobial discs, representing different classes of antimicrobial agents, were included in this study. Discs were the product of Oxoid, UK: ampicillin $(10 \mu \mathrm{g})$, amoxicillin/clavulanate $\quad(20 / 10 \quad \mu \mathrm{g})$, cefazoline $(30 \mu \mathrm{g})$, ceftazidime $(30 \mu \mathrm{g})$, ceftriaxone $(30 \mu \mathrm{g})$, ertapenem $(10 \mu \mathrm{g})$, imipenem $(10 \mu \mathrm{g})$, gentamicin $(10 \mu \mathrm{g})$, amikacin $(30 \mu \mathrm{g})$, azithromycin $(15 \mu \mathrm{g})$, levofloxacin $(5 \mu \mathrm{g})$, ciprofloxacin $(5 \mu \mathrm{g})$, trimethoprim/sulfamethoxazole $(1.25 / 23.75$ $\mu \mathrm{g})$, tetracycline $(10 \mu \mathrm{g})$, colistin $(10 \mu \mathrm{g})$, and nitrofurantoin $(300 \mu \mathrm{g})$. Isolates that showed resistance to at least three different classes of antimicrobial agents were considered as MDR. Discs were stored at $4^{\circ} \mathrm{C}$ and allowed to reach room temperature before being used. Results were calculated by measuring the inhibition zones developed around the discs in millimetre $(\mathrm{mm})$. Interpretation of results as susceptible (S), intermediate (I) or resistant (R) to a particular antimicrobial agent was performed according to CLSI (2015). For typing, isolates were grouped into different antibiotypes based on the antimicrobial susceptibility profiles (antibiograms).

\section{RAPD analysis-based genotyping}

RAPD-PCR fingerprinting was carried out to determine the genetic diversity among $K$. pneumoniae isolates.

\section{Oligonucleotide primers used in RAPD- PCR}

For RAPD analysis, preliminary PCR assays were performed to test fives primers synthesised by Eurofins Genomics, USA. Upon PCR analysis, the primer 1290 was selected based on the accuracy and reproducibility of the amplification profiles. The primers used were 10 bases long of arbitrary sequence. The details of primers used in this study are listed in Table 1.

\section{PCR reactions and cyclic conditions}

Genomic DNA was extracted from $K$. pneumoniae isolates using commercially available GeneJET Genomic DNA purification Kit (Thermo Scientific, USA). The PCR reactions were prepared in total volumes of $25 \mu \mathrm{l}$, contained $\sim 10 \mathrm{ng}$ of template DNA, 10 pmole of each primer and $12.5 \mu 1$ MyTaq HS $2 \times$ mastermix (Bioline, UK). The amplifications were done in a Veriti 96 well Thermal Cycler (Applied Biosystems, USA) programmed for $5 \mathrm{~min}$ at $94^{\circ} \mathrm{C}, 40$ cycles of denaturing at $94^{\circ} \mathrm{C}$ for 1 min, annealing at $36^{\circ} \mathrm{C}$ for $2 \mathrm{~min}$ and 
extension at $72^{\circ} \mathrm{C}$ for $2 \mathrm{~min}$, followed by a final extension at $72^{\circ} \mathrm{C}$ for $10 \mathrm{~min}$.

\section{TAE-agarose gel electrophoresis}

RAPD-PCRs products were resolved through TAE agarose gel (1 \%) electrophoresis prepared using molecular biology grade agarose (Bioline, UK) in $1 \times$ TAE buffer. DNA fragments, stained with ethedium bromide, were visualized by placing on a UV light source and photographed directly. For sizing of the separated DNA fragments, GeneRuler $1 \mathrm{~kb}$ DNA ladder (Thermo Scientific, USA) was used.

\section{RAPD profiles analysis}

RAPD patterns were analyzed and binary scoring was carried out using GelQuest computer software. UPGMA clusters showing the genetic similarity of the isolates were plotted using Numerical Taxonomy System software (NTSYS, Applied Biostatistics, Inc) based on Jaccard coefficient.

\section{Discriminatory power of typing methods}

The Simpson's diversity index was calculated to assess the discriminatory power of the biotyping, antibiogram typing and PCR-based RAPD typing methods performed in this study.

\section{Results and Discussion}

Identification and biotyping of $K$. pneumoniae isolates

A total of $43 \mathrm{~K}$. pneumoniae isolates were isolated from different clinical specimens, collected from cancer patients in this study. The 43 isolates were identified by culture characteristics, standard biochemical procedures and the identification kit API
20E; that showed the typical profiles of $K$. pneumoniae. For biotyping, the API $20 \mathrm{E}$ system identified three different biochemical profiles (or index numbers) based on the differences in the ability of $K$. pneumonia isolates to utilize different carbon sources. The biochemical profiles were arbitrarily designated as B1, B2 and B3 that have three different profiles 5215773, 7215773, and 7214773, respectively, with biotype B1 (code number 5215773) was the most frequent biotype observed in $39(91 \%)$ of $K$. pneumoniae isolates (Table 2).

Antimicrobial susceptibility patterns and antibiogram-based phenotyping of $K$. pneumoniae isolates

The antimicrobial susceptibility patterns showed higher frequencies of resistance among $K$. pneumoniae isolates. All $K$. pneumoniae isolates (100\%) were resistant to each ampicillin, amoxicillin/clavulanic acid and cephazolin, in addition to $97.7 \%$ of isolates was resistant to ertapenem and ceftriaxone, $95.3 \%$ of isolates was resistant to trimethoprim-sulfamethoxazole and ceftazidim. Colistin showed lowest level of resistance $19(44.2 \%)$ isolates. Table.3 shows the antimicrobial susceptibility of $K$. pneumoniae isolates. All $K$. pneumoniae isolates $(100 \%)$ in this study were described as MDR as all isolates showed resistance to at least three or more classes of antimicrobial agents. Based on the antibiograms, the $43 \mathrm{~K}$. pneumoniae isolates were grouped into 25 antibiogram types (antibiotypes), designated as A1 to A25, depending upon their resistance to the antimicrobial agents tested (Table.4).

PCR-based RAPD genotyping of $K$. pneumoniae isolates

$K$. pneumoniae isolates were analyzed by PCR-based RAPD fingerprinting technique using five primers (RAPD4, 640, 1247, 
1252,1290 ) with $\mathrm{G} / \mathrm{C}$ contents ranging from 50 to $80 \%$. The primer 1290 containing a 60 $\% \mathrm{G} / \mathrm{C}$ content gave a good discriminatory result. Thus, based on the fingerprint clarity and discrimination obtained, primer 1290 was used for RAPD analysis of $K$. pneumoniae isolates throughout this study. RAPD-PCR amplifications with primer 1290 resulted in DNA fragments ranging from approximately $0.27-2.7 \mathrm{kbp}$. Notably, all DNA fragments were under $3 \mathrm{kbp}$, which is typical for RAPD profiles. Although many fragments appeared common to several isolates, the patterns were qualitatively sufficient for accurate isolates differentiation. The strains were considered to be within a pattern if the level of similarity was $70 \%$ or more, thus the RAPD analysis revealed 39 distinct patterns (Figure 1B). The 39 RAPD genotypes were arbitrarily designated R1 to R39. Variant subtypes having a banding pattern similarity of $70 \%$ or more were indicated by a letter suffix. Thus, R7 had three variant patterns (R7a, R7b, R7c), while R8 and R29 had two variant patterns (R8a, R8b and R29a and R29b, respectively).

GeneRuler $1 \mathrm{~kb}$ DNA Molecular weight marker (Thermo Scientific, USA). (B) Corresponding dendrogram generated with Jaccard's coefficient and the UPGMA clustering method.

\section{Discriminatory power of typing methods}

The Simpson's diversity index for biotyping, antibiogram typing and PCR-based RAPD typing methods were $0.17,0.92$ and 0.99 , respectively, where RAPD profiles exhibited the highest discriminatory power. The discriminatory power of each typing method is shown in Table.5. Typing of all isolates included in this study is summarized in Table.6. $K$. pneumoniae is an important opportunistic pathogen causing serious hospital-acquired as well as community- acquired infections (Cryz et al., 1984; Nordmann et al., 2009). Notably, patients with underlying malignancy presenting with bacteraemia are more likely to be infected with $K$. pneumoniae. This may be explained partly by $K$. pneumoniae virulence factors, such as capsule, pili, lipopolysaccharide and siderophore, which can give an adaptive advantage in patients with underlying malignancy and increase the potential for gastrointestinal translocation or biofilm formation in indwelling intravascular catheters; thus resulting in $K$. pneumoniae bacteraemia over other intestinal colonizers (Henao-Martínez et al., 2013). In a cohort study, underlying malignancy was identified in $63 \%$ of $K$. pneumoniae nosocomial bacteraemia (Henao-Martínez et al., 2013).

The prevalence of infections caused by MDR K. pneumoniae has increased during the last decade, reflecting the selective pressure posed by the extensive and misuse of antimicrobial drugs. Thus, MDR $K$. pneumoniae is considered as an important health problem due to limited options for antimicrobial therapy resulting in higher morbidity and mortality rates (Correa et al., 2013; Hou et al., 2015). In the current study, the microbial diversity among MDR $K$. pneumoniae isolated from cancer patients was investigated using phenotypic and RAPD-based molecular typing methods for homology analyses. A total of $43 K$. pneumoniae isolates, recovered from different clinical specimens, collected from cancer patients at National Cancer Institute, Cairo, Egypt, were included in the present study. For biotyping, the biochemical system profile number of the API $20 \mathrm{E}$ system (BioMerieux, France) clustered $K$. pneumoniae isolates in this study into three biotypes designated $\mathrm{B} 1, \mathrm{~B} 2$, and $\mathrm{B} 3$ with three different biochemical profiles of 5215773, 7215773 and 7214773, respectively. The biotype $\mathrm{B} 1$ was the most 
predominant one among isolates comprise $39(90.7 \%)$ isolates, followed by B2 in three (7 \%) isolates and B3 represented by only one isolate $(2.3 \%)$. In another study, the biochemical profile 5215773 represented $37.5 \%$ among $K$. pneumoniae isolates from blood, urine and sputum (Khattak and Fraise, 2011). In addition, the antibiogram typing based on antibiograms (susceptibility patterns to different antimicrobial agents tested) obtained in this study grouped the $K$. pneumoniae isolates into 25 antibiotypes (antibiograms) designated A1 to A25.

MDR bacteria are defined as the bacteria resistant to at least one agent in three or more classes of antimicrobial agents (Magiorakos et al., 2012). Following this definition, all $K$. pneumoniae isolates included in this study were described as MDR as all $43(100 \%)$ isolates showed resistance to at least three or more classes of antimicrobial agents. $K$. pneumoniae is naturally resistant to ampicillins and early cephalosporins due to the production of the chromosomal mediated extended-spectrum $\beta$-lactamases (ESBLs) in this organism. However, the acquisition of resistance to amoxicillin/clavulanic acid and broadspectrum cephalosporins has become a global phenomenon showing variable occurrence rates worldwide (Bouzenoune et al., 2009).

The antimicrobial susceptibility testing showed highest resistance frequency of 100 $\%$ among $K$. pneumoniae isolates to each ampicillin, amoxicillin/clavulanic acid and cephazolin, followed by resistance frequencies of $97.7 \%$ and $95.3 \%$ to ceftriaxone and ceftazidim, respectively, suggesting that these drugs are unreliable for the routine treatment of $K$. pneumoniae infections in this institution. Effective antimicrobial drugs, such as aminoglycosides, fluoroquinolones and carbapenems, have been used to treat ESBLproducing $K$. pneumoniae infections (Nordmann and Mammer 2007; He et al., 2015). Although, in the current study, $K$. pneumoniae isolates showed high resistance to gentamicin and amikacin of $69.8 \%$ and $55.8 \%$, respectively. $K$. pneumoniae showed high resistance rates to the tested fluoroquinolones drugs ciprofloxacin and levofloxacin of $81.4 \%$ and $65.1 \%$, respectively. $K$. pneumoniae had intrinsic sensitivity to fluoroquinolones which is commonly used for empirical treatment of urinary tract infections. Because of extensive use of fluoroquinolones as an alternative medication to treatment failure with other routine drugs, this might be responsible for the high non-susceptibility to quinolones in $K$. pneumoniae nowadays (Nordmann and Mammer 2007). Resistance rate to ciprofloxacin was $33 \%$ in the Rabat region (Morocco) (Bouzenoune et al., 2009). Although, carbapenems have been considered as last option treatments against infections caused by MDR Gram-negative organisms. K. pneumoniae has developed an efficient carbapenem resistance mechanism, known as KPC (Klebsiella pneumoniae carbapenemase) (Naas et al., 2008). KPC enzyme-producing $K$. pneumonia is generally susceptible to few antimicrobial agents, and it is associated with a high mortality rate among patients with bloodstream infections (Vuotto et al., 2014).

In this study, carbapenem drugs including imipenem and ertapenem, showed higher resistance rates of $74.4 \%$ and $97.7 \%$, respectively. In this study, trimethoprim /sulfamethoxazole showed significant higher resistance rate of $95.3 \%$. Trimethoprim /sulfamethoxazole combination has been used extensively for the treatment of urinary tract infections (UTIs), particularly that caused by $K$. pneumoniae which led to higher resistance levels. The resistance 
profile to trimethoprim/sulfamethoxazole was reported to be $61 \%$ in Morocco (Bouzenoune et al., 2009). With growing resistance of Enterobacteriaceae to the commonly used antimicrobial agents, nitrofurantoin has become increasingly important in the treatment of UTIs. They are known to have less potential for promoting resistance and therefore should be used preferentially.

Table.1 Nucleotide sequences of oligonucleotides used in RAPD-PCR analysis

\begin{tabular}{|c|c|c|c|}
\hline Primer & Sequence 5' to 3 & GC $(\boldsymbol{\%})$ & Reference \\
\hline RAPD4 & AAGACGCCGT & 60 & Sachse et al. (2014) \\
\hline 640 & CGTGGGGCCT & 80 & Eftekhar and Nouri (2015) \\
\hline 1247 & AAGAGCCCGT & 60 & \multirow{2}{*}{ Samra et al. (2007) } \\
\hline 1252 & GCGGAAATAG & 50 & \\
\hline 1290 & GTGGATGCGA & 60 & \\
\hline
\end{tabular}

Table.2 Biotypes of K. pneumoniae isolates based on API 20E analytical profiles.

\begin{tabular}{|c|c|c|}
\hline Biotype & Code No. & No. of isolates (\%) \\
\hline B1 & 5215773 & $39(90.7)$ \\
\hline B2 & 7215773 & $3(7)$ \\
\hline B3 & 7214773 & $1(2.3)$ \\
\hline
\end{tabular}

*Percentage correlated to the total number of isolates.

Table.3 Frequency of the antimicrobial susceptibilities among $K$. pneumoniae isolates.

\begin{tabular}{|l|c|c|}
\hline \multicolumn{1}{|c|}{ Antimicrobial Agent } & $\begin{array}{c}\text { Sensitive } \\
\text { No. of isolates }\left(\boldsymbol{\%}^{\mathbf{1}}\right)\end{array}$ & $\begin{array}{c}\text { Resistant } \\
\text { No. of isolates }\left(\boldsymbol{\%}^{\mathbf{2}}\right)\end{array}$ \\
\hline Colistin (CT) & $24(55.8)$ & $19(44.2)$ \\
\hline Amikacin (AK) & $19(44.2)$ & $24(55.8)$ \\
\hline Gentamycin (CN) & $13(30.2)$ & $30(69.8)$ \\
\hline Ciprofloxacin (CIP) & $8(18.6)$ & $35(81.4)$ \\
\hline Levofloxacin (LEV) & $15(34.9)$ & $28(65.1)$ \\
\hline Tertracycline (TE) & $11(25.6)$ & $32(74.4)$ \\
\hline Azithromycin (AZM) & $6(14)$ & $37(86)$ \\
\hline Nitrofurantoin (F) & $5(11.6)$ & $38(88.4)$ \\
\hline Trimethoprim/sulfamethoxazole (SXT) & $2(4.7)$ & $41(95.3)$ \\
\hline Imipenem (IMP) & $11(25.6)$ & $32(74.4)$ \\
\hline Ertapenem (ETP) & $1(2.3)$ & $42(97.7)$ \\
\hline Ceftazidime (CAZ) & $2(4.7)$ & $41(95.3)$ \\
\hline Ceftriaxone (CRO) & $1(2.3)$ & $42(97.7)$ \\
\hline Ampicillin (AMP) & $0(0)$ & $43(100)$ \\
\hline Amoxicillin/clavulanic acid (AMC) & $0(0)$ & $43(100)$ \\
\hline Cephazolin (KZ) & $0(0)$ & $43(100)$ \\
\hline 1,2Percentages correlated to the total number of isolates.
\end{tabular}


Table.4 Antibiotypes of $K$. pneumoniae isolates based on antibiogram patterns.

\begin{tabular}{|c|c|c|}
\hline Antibiotype & Antibiogram pattern & $\begin{array}{c}\text { No. of isolates } \\
(\%) *\end{array}$ \\
\hline A1 & Resistant to all antimicrobial agent classes tested & $10(23)$ \\
\hline $\mathbf{A 2}$ & Resistant to all antimicrobial agent classes used except CT & $7(16)$ \\
\hline $\mathbf{A 3}$ & Resistant to all antimicrobial agent classes used except $\mathrm{CN}$ & $1(2)$ \\
\hline A4 & Resistant to all antimicrobial agent classes used except CT and AK & $3(7)$ \\
\hline A5 & Resistant to all antimicrobial agent classes used except $\mathrm{CT}$ and $\mathrm{CN}$ & $1(2)$ \\
\hline A6 & Resistant to all antimicrobial agent classes used except CT and TE & $2(5)$ \\
\hline A7 & Resistant to all antimicrobial agent classes used except CT and AZM & $1(2)$ \\
\hline A8 & Resistant to all antimicrobial agent classes used except CN, AK and LEV & $1(2)$ \\
\hline A9 & Resistant to all antimicrobial agent classes used except CN, LEV and IMP & $1(2)$ \\
\hline A10 & Resistant to all antimicrobial agent classes used except CT, AK, LEV and F & $1(2)$ \\
\hline A11 & Resistant to all antimicrobial agent classes used except CT, AK, TE and AMP & $1(2)$ \\
\hline A12 & Resistant to all antimicrobial agent classes used except CT, CN, AK and LEV & $1(2)$ \\
\hline $\mathbf{A 1 3}$ & Resistant to all antimicrobial agent classes used except CT, CN, AZM and F & $1(2)$ \\
\hline A14 & Resistant to all antimicrobial agent classes used except AK, TE, LEV, IMP, and CIP & $1(2)$ \\
\hline A15 & Resistant to all antimicrobial agent classes used except AK, TE, LEV, IMP, and F & $1(2)$ \\
\hline A16 & Resistant to all antimicrobial agent classes used except CN, AK, LEV, IMP, and CIP & $1(2)$ \\
\hline A17 & Resistant to all antimicrobial agent classes used except CT, CN, AK, TE, and AZM & $1(2)$ \\
\hline A18 & $\begin{array}{l}\text { Resistant to all antimicrobial agent classes used except CN, AK, LEV, AZM, CAZ and } \\
\text { SXT }\end{array}$ & $1(2)$ \\
\hline A19 & Resistant to all antimicrobial agent classes used except CT, AK, LEV, IMP, CIP, and F & $1(2)$ \\
\hline A20 & $\begin{array}{l}\text { Resistant to all antimicrobial agent classes used except CN, AK, TE, LEV, IMP and } \\
\text { CIP }\end{array}$ & $1(2)$ \\
\hline A21 & Resistant to all antimicrobial agent classes used except CT, AK, TE, LEV, IMP, and F & $1(2)$ \\
\hline A22 & Resistant to all antimicrobial agent classes used except CT, AK, TE, LEV, IMP and CIP & $1(2)$ \\
\hline $\mathbf{A 2 3}$ & $\begin{array}{l}\text { Resistant to all antimicrobial agent classes used except CT, CN, AK, TE, LEV, IMP and } \\
\text { CIP }\end{array}$ & $1(2)$ \\
\hline A24 & $\begin{array}{l}\text { Resistant to all antimicrobial agent classes used except CT, CN, AK, LEV, IMP, CIP, } \\
\text { AZM and ETP }\end{array}$ & $1(2)$ \\
\hline A25 & $\begin{array}{l}\text { Sensitive to all antimicrobial agent classes used except AMP, ETP, F, CT, KZ and } \\
\text { AMC }\end{array}$ & $1(2)$ \\
\hline
\end{tabular}

"Percentages correlated to the total number of isolates. AMP, ampicillin; CT, colistin; CN, gentamicin; AK, amikacin; TE, tetracycline; AZM, azithromycin; LEV, levofloxacin; IMP, imipenem; F, nitrofurantoin; AMP, ampicillin; CIP, ciprofloxacin; SXT, trimethoprim-sulfamethoxazole; CAZ, ceftazidime; ETP, ertapenem; AMC, Amoxacillin/clavulanic acid; KZ, cephazolin.

Table.5 Simpons's index of diversity for K. pneumoniae isolates in this study.

\begin{tabular}{ccc}
\hline Typing method & No. of diverse types & Simpons's index of diversity \\
\hline RAPD-PCR & 39 & 0.9945 \\
Antibiogram typing & 25 & 0.9225 \\
Biotyping & 3 & 0.176 \\
\hline
\end{tabular}


Table.6 Summary of biotyping, antibiogram typing (antibiotyping) and RAPD-PCR typing of $K$. pneumoniae isolates relative to source of specimens.

\begin{tabular}{|c|c|c|c|c|}
\hline \multirow{2}{*}{ Specimen } & \multirow{2}{*}{$\begin{array}{c}\text { No. of } \\
\text { isolates }\end{array}$} & \multicolumn{2}{|c|}{$\begin{array}{c}\text { Phenotype } \\
\end{array}$} & \multirow{2}{*}{ RAPD-based genotype (No.)* } \\
\hline & & Biotype (No.)* & Antibiotype (No.)* & \\
\hline Blood & 27 & $\begin{array}{l}\text { B1 (24), B2 } \\
(2), \text { B3 (1) }\end{array}$ & $\begin{array}{l}\text { A1 (2), A2 (4), A3 (1), } \\
\text { A4 (3), A5 (1), A6 (1), } \\
\text { A9 (1), A10 (1), A12 } \\
(1), \text { A13 (1), A14 (1), } \\
\text { A15 (1), A16 (1), A17 } \\
(1), \text { A18 (1), A19 (1), } \\
\text { A22 (1) A23, (1), A24 } \\
(1), \text { A25 (1) }\end{array}$ & $\begin{array}{l}\text { R4 (1), R5 (1), R6 (1), R7a (1), } \\
\text { R7c (1), R8a (1), R8b (1), R21 } \\
(1), \text { R22 (1), R23 (1), R24 (1), } \\
\text { R25 (1), R26 (1), R27 (1), R28 } \\
(1), \text { R29a (1), R29b (1), R30 } \\
\text { (1), R31 (1), R32 (1), R33 (1), } \\
\text { R34 (1), R35 (1), R36 (1), R37 } \\
\text { (1), R38 (1), R39 (1) }\end{array}$ \\
\hline Pus & 10 & B1 (9), B2 (1) & $\begin{array}{l}\text { A1 (4), A6 (1), A7 (1), } \\
\text { A8 (1), A11 (1), A20 } \\
(1), \text { A21 (1) }\end{array}$ & $\begin{array}{l}\text { R1 (1), R2 (1), R3 (1), R7b } \\
\text { (1), R9 (1), R10 (1), R11 (1), } \\
\text { R12 (1), R13 (1), R15 (1) }\end{array}$ \\
\hline Sputum & 4 & B1 (4) & $\mathrm{A} 1(2), \mathrm{A} 2(4)$ & $\begin{array}{l}\text { R16 (1), R17 (1), R18 (1), R19 } \\
\text { (1) }\end{array}$ \\
\hline Stool & 1 & $\mathrm{~B} 1(1)$ & A2 (1) & R14 (1) \\
\hline Urine & 1 & $\mathrm{~B} 1(1)$ & A1 (1) & R20 (1) \\
\hline
\end{tabular}

*No. of isolates represent each type.

Fig.1 PCR-based RADP RAPD patterns of K. pneumoniae isolates with primer 1290. (A) Agarose gel (1\%) electrophoresis of amplification products; GeneRuler 1 kb DNA Molecular weight marker (Thermo Scientific, USA).

\section{(A)}
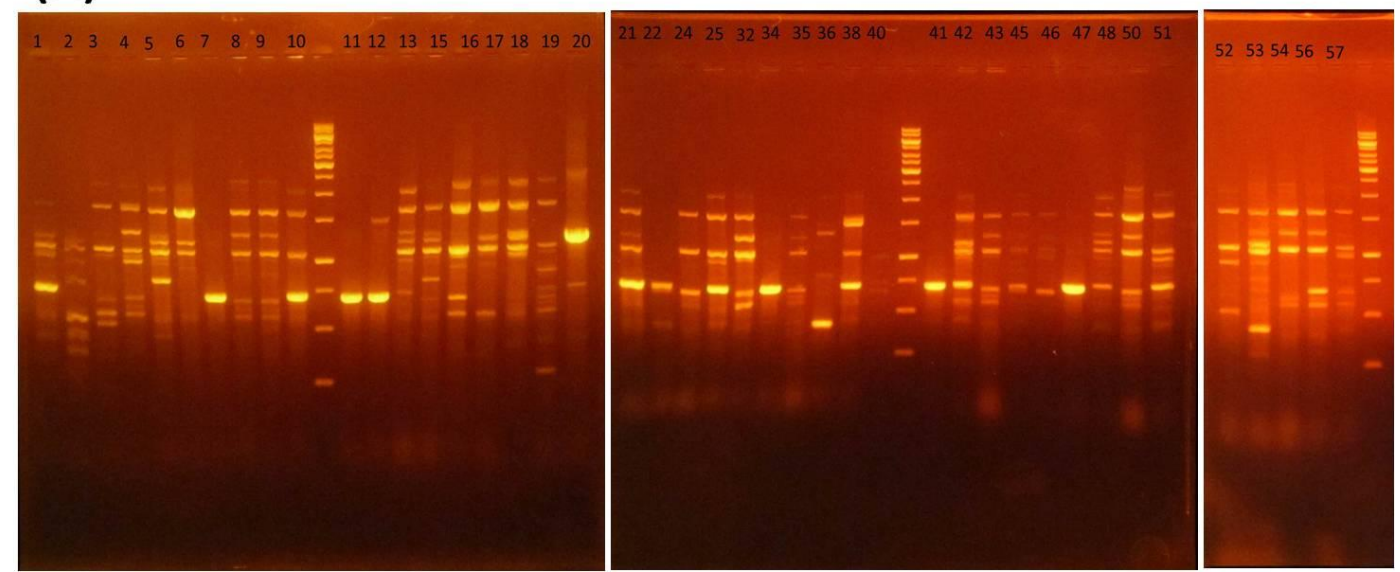
(B) Corresponding dendrogram generated with Jaccard's coefficient and the UPGMA clustering method.

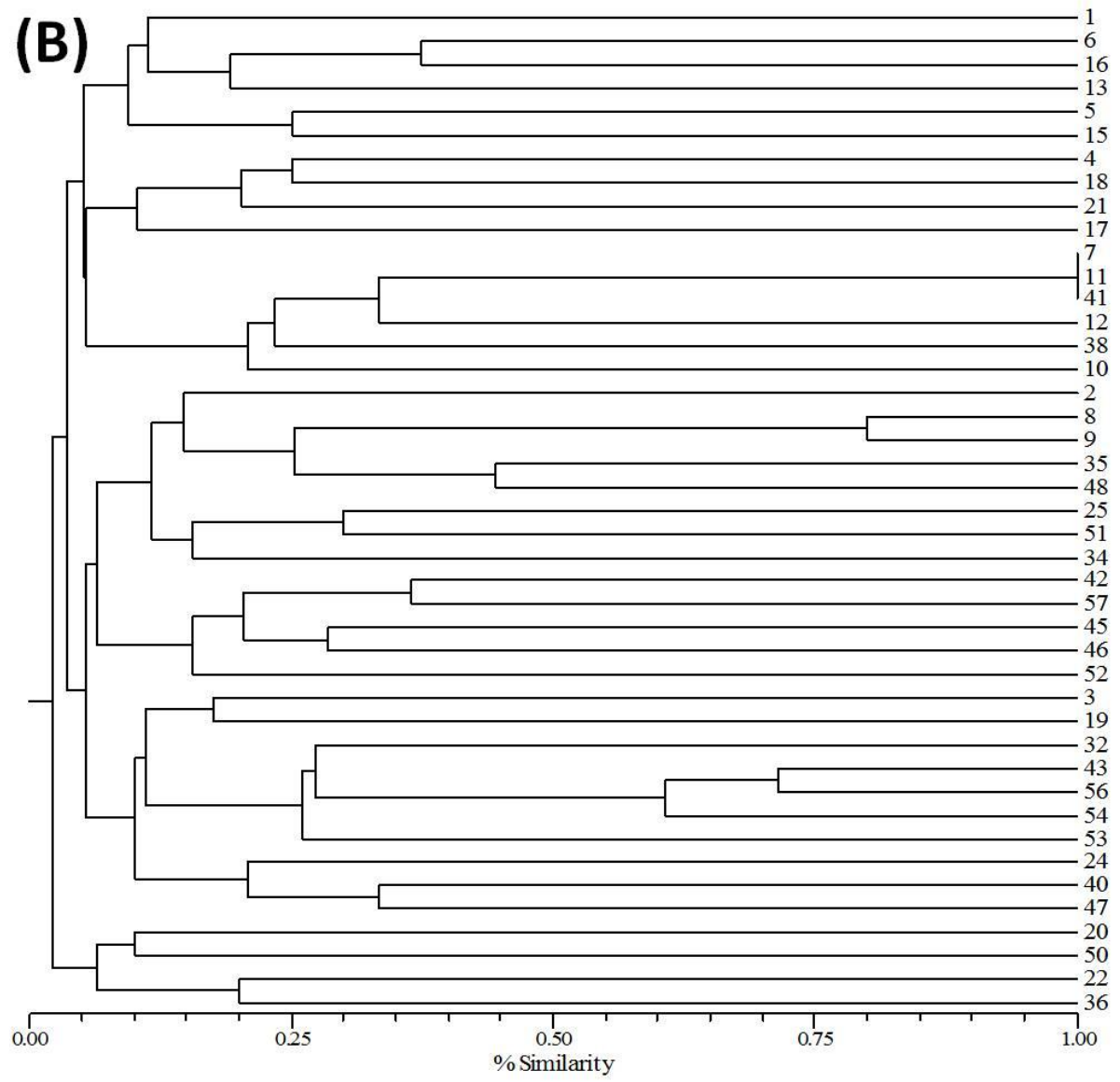

However, the susceptibility of $K$. pneumoniae isolates in this study to nitrofurantoin was low $(11.6 \%)$. Although tetracyclines have decreased susceptibility to develop resistance (Pieboji et al., 2004), in our study $74.4 \%$ of our isolates were nonsusceptible to tetracyclines. These findings of increasing the resistance profiles to tested antimicrobials suggested that these antimicrobial agents may not be appropriate for initiation of empirical therapy of infections caused by $K$. pneumoniae in this institution of study and/or in a developing country like Egypt. However, the highest susceptibility profile was shown in this study to colistin as $55.8 \%$ of isolates were sensitive, which could be attributed to the limited use of this antimicrobial agent and may suggest using it in the empirical therapy for $K$. pneumoniae infections.

In the current study, the genetic diversity of $K$. pneumoniae isolates with similar biotypes and/or multidrug resistance profiles was investigated using arbitrarily primed RAPD analysis. The RAPD patterns obtained was clustered by dendrogram generated with Jaccard's coefficient and the UPGMA clustering method. PCR-based RAPD fingerprinting of the $43 \mathrm{~K}$. pneumoniae isolates revealed a significant molecular heterogeneity of $K$. pneumoniae isolated within this hospital indicated by 39 different RAPD-based groups designated R1 to R39 
and/or 43 different patterns were observed among isolates. That demonstrates the high discriminatory power of RAPD with the primer 1290. Fortunately, the existence of all isolates within distinguished RAPD patterns indicated that there was no occurrence of bacterial spread among patients. In addition, pathogenic $K$. pneumoniae isolated from the institute comprise a genetically variable group of organisms. These results are consistent with Lai et al., (2000) observation that pathogenic $K$. pneumoniae population is highly heterogeneous, based on the distribution of different nucleotide sequences.

The biochemical profiles and antibiograms are usually not reliable to show microbial diversity and more discriminatory epidemiological marker, such as molecular methods, should be used for microbial typing (Limansky et al., 2004). In the current study, the Simpons's index of diversity showed a higher discriminatory power of PCR-based RAPD (0.995) over antibiogram typing (0.922) and biotyping (0.176). Although, the antibiogram typing showed an outstanding discriminatory power comparable to that of PCR-based RAPD; suggesting the possibility of combining the two methods for detection of microbial diversity of $K$. pneumoniae isolates. The epidemiological investigation based on APIbased biotyping may not accurately predict relatedness of the strains as shown in the present study. Although, both biotyping and antibiogram typing of $K$. pneumoniae isolates could be well for preliminary screening of strain relatedness.

In conclusion, in this study, MDR $K$. pneumoniae is becoming a serious problem in cancer patients due to limited choice for treatment. The epidemiological typing of 43 MDR $K$. pneumoniae clinical isolates was performed using phenotypic and molecular typing methods. $K$. pneumoniae infections in this institute were caused by a variety of bacterial genotypes and/or phenotypes. However, RAPD clearly prevailed among the other typing methods (biotyping, antibiotyping) and proved to be a useful technique in distinguishing related and unrelated $K$. pneumoniae clinical isolates. Continuous studies should be carried out to investigate the antimicrobial resistance and to try the use of antimicrobial combinations to overcome these resistances. We propose that infection control measures and strict antimicrobial stewardship policies should be applied to reduce the selective pressure that favours the emergence and epidemic of MDR bacteria.

\section{References}

Ashayeri-Panah, M., Feizabadi, M.M. and Eftekhar, F. 2014. Correlation of Multi-drug Resistance, Integron and bla ESBL Gene Carriage with Genetic Fingerprints of Extended-Spectrum $\beta$ Lactamase Producing Klebsiella pneumoniae. Jundishapur Microbiol., 7(2).

Bouzenoune, F., Boudersa, F., Bensaad, A., Harkat, F. and Siad, N. 2009. Urinary tract infections in Ain M'lila (Algeria). Antibiotic resistance of 239 strains isolated between 2006 and 2007. Médecine et Maladies Infectieuses, 39(2): 142-143.

Cao, F., Wang, X., Wang, L., Li, Z., Che, J., Wang, L., Li, X., Cao, Z., Zhang, J., Jin, L. and $\mathrm{Xu}, \mathrm{Y}$. 2015. Evaluation of the efficacy of a bacteriophage in the treatment of pneumonia induced by multidrug resistance Klebsiella pneumoniae in mice. BioMed. Res. Int., 24.

Cartelle, M., del Mar Tomas, M., Pertega, S., Beceiro, A., Dominguez, M.A., Velasco, D., Molina, F., Villanueva, 
R. and Bou, G. 2004. Risk factors for colonization and infection in a hospital outbreak caused by a strain of Klebsiella pneumoniae with reduced susceptibility to expanded-spectrum cephalosporins. $J$. Clin. Microbiol., 42(9): 4242-4249.

CLSI. 2015. Performance Standards for Antimicrobial Susceptibility Testing; $25^{\text {th }}$ informational Supplement. CLSI document M100-S24. Pages: 44-50.

Correa, L., Martino, M.D.V., Siqueira, I., Pasternak, J., Gales, A.C., Silva, C.V., Camargo, T.Z.S., Scherer, P.F. and Marra, A.R. 2013. A hospital-based matched case-control study to identify clinical outcome and risk factors associated with carbapenem-resistant Klebsiella pneumoniae infection. $B M C$ Infect. Dis., 13(1): 80.

Cryz, S.J., Fürer, E. and Germanier, R. 1984. Protection against fatal Klebsiella pneumoniae burn wound sepsis by passive transfer of anticapsular polysaccharide. Infect. Immunity, 45(1): 139-142.

Eftekhar, F. and Nouri, P. 2015. Correlation of RAPD-PCR Profiles with ESBL Production in Clinical Isolates of Klebsiella pneumoniae in Tehran. $J$. Clin. Diag. Res., JCDR, 9(1): DC01.

Filgona, J., Banerjee, T. and Anupurba, S. 2015. Role of efflux pumps inhibitor in decreasing antibiotic resistance of Klebsiella pneumoniae in a tertiary hospital in North India. J. Infect. Develop. Countries, 9(08): 815-820.

He, F., Fu, Y., Chen, Q., Ruan, Z., Hua, X., Zhou, H. and Yu, Y. 2015. Tigecycline susceptibility and the role of efflux pumps in tigecycline resistance in KPC-producing Klebsiella pneumoniae. PloS one, 10(3): e0119064.

Henao-Martínez, A.F., González-Fontal, G.R., Castillo-Mancilla, J.R. and
Yang, I.V. 2013. Enterobacteriaceae bacteremias among cancer patients: an observational cohort study. Int. J. Infect. Dis., 17(6): e374-e378.

Holt, K.E., Wertheim, H., Zadoks, R.N., Baker, S., Whitehouse, C.A., Dance, D., Jenney, A., Connor, T.R., Hsu, L.Y., Severin, J. and Brisse, S. 2015. Genomic analysis of diversity, population structure, virulence, and antimicrobial resistance in Klebsiella pneumoniae, an urgent threat to public health. Proceedings of the National Academy of Sciences, 112(27): E3574E3581.

Hou, X.H., Song, X.Y., Ma, X.B., Zhang, S.Y. and Zhang, J.Q. 2015. Molecular characterization of multidrug-resistant Klebsiella pneumoniae isolates. Brazilian J. Microbiol., 46(3): 759768.

Khattak, M.N. and Fraise, A. 2011. Clonal spread of multi-drug resistant Klebsiella pneumoniae isolates in a large teaching hospital in the UK. $J$. Postgraduate Med. Institute (Peshawar-Pakistan), 19(2): 130-134.

Lai, Y.C., Yang, S.L., Peng, H.L. and Chang, H.Y. 2000. Identification of genes present specifically in a virulent strain of Klebsiella pneumoniae. Infect. immunity, 68(12): 7149-7151.

Li, W., Raoult, D. and Fournier, P.E. 2009. Bacterial strain typing in the genomic era. FEMS Microbiol. Rev., 33(5): 892-916.

Limansky, A.S., Zamboni, M.I., Guardati, M.C., Rossignol, G., Campos, E. and Viale, A.M. 2004. Evaluation of phenotypic and genotypic markers for clinical strains of Acinetobacter baumannii. MEDICINA-BUENOS AIRES, 64: 306-312.

Magiorakos, A.P., Srinivasan, A., Carey, R.B., Carmeli, Y., Falagas, M.E., Giske, C.G., Harbarth, S., Hindler, 
J.F., Kahlmeter, G., Olsson-Liljequist, B. and Paterson, D.L. 2012. Multidrug-resistant, extensively drug-resistant and pandrug-resistant bacteria: an international expert proposal for interim standard definitions for acquired resistance. Clin. Microbiol. Infect., 18(3): 268-281.

Naas, T., Cuzon, G., Villegas, M.V., Lartigue, M.F., Quinn, J.P. and Nordmann, P. 2008. Genetic structures at the origin of acquisition of the $\beta$ lactamase blaKPC gene. Antimicrob. Agents And Chemother., 52(4): 12571263.

Nordmann, P., Cuzon, G. and Naas, T. 2009. The real threat of Klebsiella pneumoniae carbapenemase-producing bacteria. The Lancet Infect. Dis., 9(4): 228-236.

Nordmann, P. and Mammeri, H. 2007. Résistance plasmidique aux quinolones. Antibiotiques, 9(4): 246253.

Passet, V. and Brisse, S. 2015. Associatio of tellurite resistance with hypervirulent clonal groups of Klebsiella pneumoniae.J. Clin. Microbiol., 53(4): 1380-1382.

Sachse, S., Bresan, S., Erhard, M., Edel, B., Pfister, W., Saupe, A. and Rödel, J.
2014. Comparison of multilocus sequence typing, RAPD, and MALDITOF mass spectrometry for typing of $\beta$-lactam-resistant Klebsiella pneumoniae strains. Diag. Microbiol. Infect. Dis., 80(4): 267-271.

Samra, Z., Ofir, O., Lishtzinsky, Y., MadarShapiro, L. and Bishara, J. 2007. Outbreak of carbapenem-resistant Klebsiella pneumoniae producing KPC-3 in a tertiary medical centre in Israel. Int. J. Antimicrobial Agents, 30(6): 525-529.

Tan, H.L., Wang, Y., Cheng, X.Q., Huang, Y.M., Liu, W. and Zhang, L.J. 2015. Genome sequence of an extensively drug-resistant strain of Klebsiella pneumoniae, strain $\mathrm{YN}-1$, with carbapenem resistance. Genome announcements, 3(1): e01279-14.

Vuotto, C., Longo, F., Balice, M.P., Donelli, G., Varaldo, P.E. 2014. Antibiotic resistance related to biofilm formation in Klebsiella pneumoniae. Pathogens, 3: 743-58.

Zhao, F., Bai, J., Wu, J., Liu, J., Zhou, M., Xia, S., Wang, S., Yao, X., Yi, H., Lin, M. and Gao, S. 2010. Sequencing and genetic variation of multidrug resistance plasmids in Klebsiella pneumoniae. Plos One, 5(4): e10141.

\section{How to cite this article:}

Mahmoud M. Tawfick, Samira M. Hamed, Hadeel M. Darwich and Hadir A. El-Mahallawy. 2016. Phenotypic and Genotypic Diversity of Nosocomial Multi-Drug Resistant Klebsiella pneumoniae Isolated from Cancer Patients in Cairo, Egypt. Int.J.Curr.Microbiol.App.Sci. 5(7): 931-943. doi: http://dx.doi.org/10.20546/ijcmas.2016.507.105 